\title{
Three cases of ocular syphilis and the resurgence of the disease in Queensland
}

This article was published in the following Dove Press journal:

International Medical Case Reports Journal

9 September 2016

Number of times this article has been viewed

\author{
Sergio A Sara \\ Andrew S McAllister \\ Department of Ophthalmology, Gold \\ Coast University Hospital (GCUH), \\ Southport, QLD, Australia
}

\begin{abstract}
The past few years has seen a resurgence of syphilis. It is predominantly associated within men who have sex with men and also within heterosexual Indigenous Australians. Possessing the ability to mimic a variety of ocular diseases, it typically manifests as uveitis, although it can affect any structure within the eye. Thus, a high degree of clinical suspicion by ophthalmologists is required to prevent disease progression and ocular morbidity. Patients require prolonged antibiotic treatment with intravenous benzylpenicillin and outpatient monitoring to successfully resolve the infection. We describe a case series of ocular syphilis presentations in Queensland, Australia.
\end{abstract}

Keywords: ocular syphilis, Indigenous Australians, men who have sex with men

\section{Background}

Syphilis is a sexually transmitted infection (STI) caused by the Gram-negative spirochete Treponema pallidum. If untreated, it manifests as a chronic multisystem disease, which includes ocular morbidity. With effective antibiotic treatment since the mid-20th century, its incidence had reduced, reaching its lowest levels in early $2000 .{ }^{1}$ However, recent years have seen a resurgence of the disease, coinciding with the new era of human immunodeficiency virus (HIV) coinfection, within men who have sex with men (MSM), and within heterosexual Indigenous (Aboriginal and/or Torres Strait Islander) Australians in remote areas. ${ }^{1-3}$

Known as the "Great Masquerader", ocular syphilis has the potential to mimic a variety of ocular diseases, most commonly uveitis, but also interstitial keratitis, chorioretinitis, retinitis, retinal vasculitis, and optic and cranial neuropathies. ${ }^{4}$ It presents a diagnostic challenge for ophthalmologists, requiring a high level of clinical suspicion to avoid misdiagnosis and delay in therapy. Compounding the issue is the rise in HIV coinfection, which may equate to more severe ocular inflammation and higher rate of progression to neurosyphilis, the most devastating sequelae of syphilis. ${ }^{1,5,6}$

Despite being a rare manifestation of the disease, all routine investigative screening methodologies for ocular inflammation should include syphilis (by means of treponemal-specific and nontreponemal serology) and HIV testing. Once diagnosed, exclusion of the more serious complications arising from syphilitic infections, such as neurosyphilis and cardiovascular compromise, should be undertaken. Ocular syphilis is managed as per treatment protocol for neurosyphilis, namely, penicillin G treatment, with patients requiring prolonged outpatient follow-up..$^{1,5,7}$
Department of Ophthalmology, Gold

Coast University Hospital (GCUH),

I Hospital Blvd, Southport, QLD

4215, Australia

Email sergio.sara@health.qld.gov.au 


\section{Case reports}

All patients provided written informed consent to publish their medical data/images in this case series.

\section{Case I}

A 61-year-old male presented with his first episode of right-sided uveitis in September 2011. He was seen by an ophthalmologist and was started on topical steroids and mydriatics. His condition progressed to panuveitis, requiring subsequent vitrectomy in March 2012, with possible fungal contamination of vitreous fluid noted intraoperatively. His contralateral left eye subsequently developed vitritis in June 2012, requiring vitrectomy and intravitreal triamcinolone injection. Although potentially a contaminant, a diagnosis of HSV retinal necrosis was made from positive vitreous aspirate serology, and so he was started on oral acyclovir.

With worsening uveitis, he was eventually referred to the Townsville Hospital with a presenting visual acuity of 6/120 in the right eye, and no light perception and a relative afferent pupillary defect in the left. He was admitted and started on intravenous (IV) acyclovir. Repeat vitreous aspirate excluded HSV1/2, VZV, and toxoplasmosis and returned negative microbial studies. A previous metallic foreign body in the lower lid precluded obtaining magnetic resonance imaging studies. Repeat screening serology returned positive syphilis finding (Angiotensin converting enzyme, 18; rapid plasma reagin

A

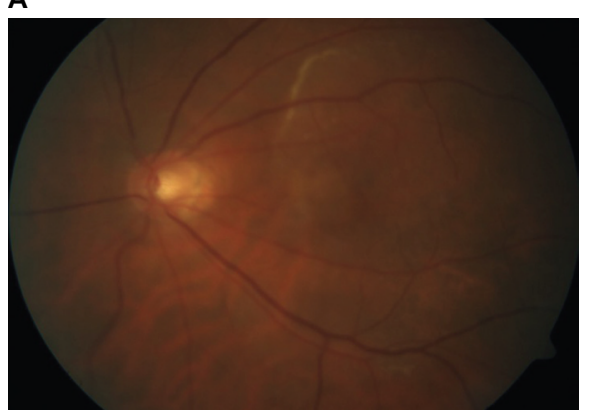

C

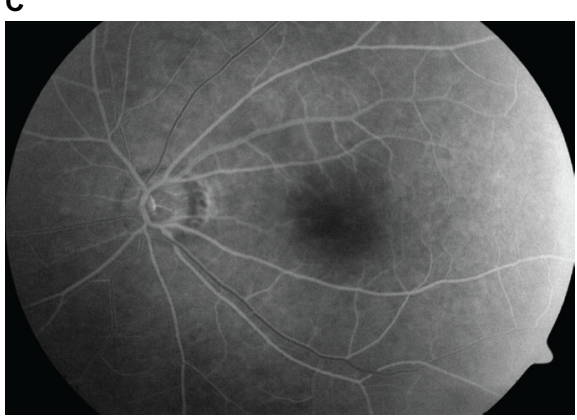

[RPR], reactive 1:128; Treponema pallidum particle agglutination assay [TPPA] and enzyme immunoassay, reactive) with subsequent negative STI screening, including HIV. He was transferred under the care of physicians and started antibiotic treatment for syphilis. Lumbar puncture (LP) and echocardiogram (ECHO) findings excluded further cardiovascular and neurological syphilitic sequelae.

He was reviewed in the outpatient department 2 weeks after his initial presentation to Townsville Hospital and diagnosed with a right total retinal detachment, requiring a vitrectomy with oil and scleral buckle. Postsurgical followup demonstrated a visual acuity of 6/60 in the right and no perception to light in the left eye.

\section{Case 2}

An otherwise healthy 74-year-old male presented to the emergency department at Townsville Hospital, with an acute episode of decreased left central vision, having a visual acuity of counting fingers in the left and of 6/7.5 in the right. Bilateral scotomas to confrontation and a localized creamy white lesion of his left macula were clinically noted. Humphrey visual field identified a right arcuate and left central defect with reduced reliability. With cilioretinal artery occlusion as a differential, fundus fluorescein angiography was performed, identifying disk hyperfluorescence and possible infiltrative/compressive lesions of the left eye (Figure 1). Further

B

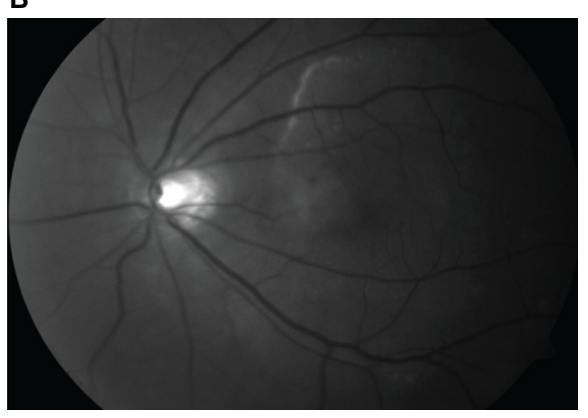

D

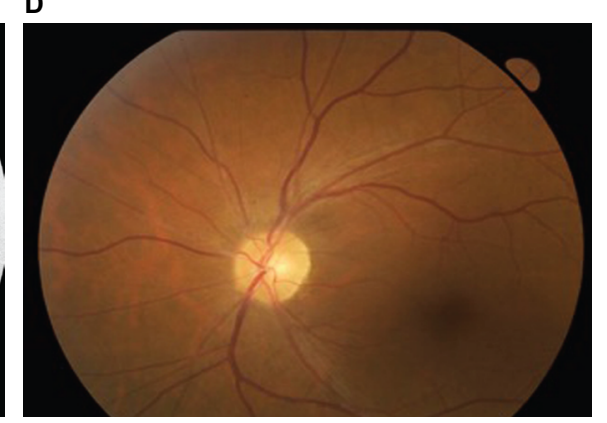

Figure I Left fundus photographs of a 74-year-old male.

Notes: (A) Fundus at presentation showing a creamy-white lesion above the superior arcade and extending to involve the macular. (B) Red-free filter of the same image allowing visual enhancement of the lesion. (C) Venous phase angiogram identifying disk hyperfluorescence. (D) Fundus photograph of the same eye following treatment completion with IV benzylpenicillin.

Abbreviation: IV, intravenous. 
investigations for optic neuritis were ordered, including a computed tomography angiography head (an magnetic resonance imaging was discounted because of a history of a spinal stimulator). Radiological imaging was normal and screening results returned positive syphilis serology ( angiotensin converting enzyme, 40; RPR, reactive $>1: 512$; TPPA and enzyme immunoassay, reactive) with negative STI screening, which included HIV. He was admitted under the medical team for extended IV benzylpenicillin treatment. Sexual history enquiries made revealed previous MSM behavior. An LP was not performed, but an ECHO demonstrated an ejection fraction of $58 \%$ with a dilated ascending aorta; subsequent computed tomography angiography confirmed aortic dilation with no aneurysm. Ophthalmology follow-up 4 months after initial presentation demonstrated improved visual acuity (right, 6/7.5; left, 6/23 improving to $6 / 9$ with pinhole) and visual field defect following completion of syphilis treatment.

\section{Case 3}

A 49-year-old HIV positive male presented with a 2 -week history of sudden-onset left central scotoma, associated with a 2-month history of left temporal photopsia. His left visual acuity on initial presentation was noted to be 6/90. On optical coherence tomography, diffuse disruption of the left retinal pigment epithelial layer, which was associated with mild vitritis and two focal pale subretinal lesions clinically, was noted. His HIV diagnosis was made 3 years earlier, likely secondary to heterosexual infection (denied IV drug use or MSM-related behaviors), and he was on highly active antiretroviral therapy, maintaining a preserved CD4 count of $>400$ on presentation. He was also in remission following chemotherapy and surgical treatment for diffuse large B-cell lymphoma.

A clinical suspicion of syphilitic retinitis was made, and screening blood for chorioretinitis returned positive syphilis serology (RPR, reactive 1:128; TPPA, reactive). He was admitted under infectious diseases for treatment of ocular syphilis, completing an extended 2-week course of IV benzylpenicillin. He was reviewed 5 weeks later in the ophthalmology outpatient department, demonstrating complete resolution of clinical findings and a returned left visual acuity of $6 / 6$.

\section{Discussion}

Although the incidence of syphilis is highest in developing nations, accounting for $90 \%$ of all new syphilis cases, the reducing prevalence in developed countries has been attributable to the use of antibiotics, notably penicillin. ${ }^{4}$
However, since 2000, the worldwide incidence of syphilis has been on a steady rise; the highest rate primarily among MSM, and recent outbreaks involve coinfection with HIV (reported to be as high as 70\%). ${ }^{1}$ The incidence of syphilis in Australia is also increasing, with current levels being the highest in recorded history. ${ }^{3}$ In line with worldwide trends, syphilitic infection in Australia is primarily observed in MSM (accounting for $89 \%$ of new diagnoses), with a threefold increasing trend seen over the past 10 years, currently 15.9 per 100,000 (2014). ${ }^{3}$ It is also highly prevalent among heterosexual Aboriginal and Torres Strait Islander (Indigenous) people in remote Australia, who have a fourfold higher rate compared to non-Indigenous Australians and an overall incidence of 49 per 100,000 (2014). ${ }^{2,3}$ In accordance with ongoing outbreaks in remote Western Australia, Northern Territory, and Queensland, levels have almost tripled among Indigenous children aged 15-19 years in the past 4 years. ${ }^{2}$ Furthermore, the rate of syphilis among MSM was the highest in HIV-confected individuals ( $8 \%$ ) compared to HIV-negative men $(2 \%){ }^{3}$

The resurgence of syphilis in recent years has also seen a global increased reporting of ocular syphilis. ${ }^{8}$ Known as the great masquerader, syphilis can involve all ocular structures throughout the different stages of infection, although typically occurs during the secondary or latent stage. ${ }^{8,9}$ Initial presentation to the ophthalmologist may include nonspecific symptoms of headache, eye pain, rash, with an elevated erythrocyte sedimentation rate, and no subsequent response to prednisolone. ${ }^{10}$ Despite its low incidence, ocular syphilis may represent the only systemic manifestation of syphilitic disease, with failure of prompt diagnosis and treatment leading to possible further transmission of a highly contagious infection, poor visual outcome, and severe neurological compromise with progression to neurosyphilis. ${ }^{8}$

The most common ocular disorder in acquired syphilis is uveitis ( $5 \%$ cases), comprising anterior uveitis, posterior uveitis, panuveitis, and/or keratouveitis. ${ }^{11,12}$ Recent literature has suggested that the main features constituting ocular syphilis are nongranulomatous inflammation, with posterior segment involvement (commonly vitritis, but also necrotizing retinitis, with serous retinal detachment; posterior placoid chorioretinitis, with peripapillary areas of subretinal pallor and acute retinal necrosis, papillitis, and retinal vasculitis). ${ }^{8,13,14}$ Poor visual prognosis has previously been reported in cases associated with sequelae, resulting in retinal damage (such as occlusive retinal vasculitis) and prolonged intraocular inflammation (such as posterior synechiae and cataracts). ${ }^{15}$ 
Poor visual prognosis may also arise from delayed treatment and diagnosis; patients with acute peripheral retinal necrosis and vasculitis may be inappropriately treated with antiviral therapy. ${ }^{9}$ Finally, syphilitic uveitis is commonly associated with elevated intraocular pressure; syphilis is one of the leading causes of secondary glaucoma in uveitis. ${ }^{9,15}$

Ocular syphilis may also present with optic nerve involvement, atrophy, inflammatory disk swelling, papilledema, and gummatous optic disk. ${ }^{9,15}$ Interestingly, optic disk swelling has been reported in $13 \%$ of patients with posterior segment involvement. ${ }^{14,15}$ Bilateral optic nerve involvement (tabes optica) with progressive binasal hemianopia has been described. ${ }^{16}$ Stromal keratitis, scleritis, and episcleritis have also been documented to occur. ${ }^{17,18}$

Given its masquerading capabilities, ophthalmologists require a high degree of clinical suspicion to screen for signs and symptoms of syphilis. ${ }^{19}$ Serologic testing for syphilis (treponemal and nontreponemal) should occur particularly if ocular inflammation has unusual characteristics, if inflammation is severe with treatment delay likely to lead to further damage, for a positive sexual history or high-risk patient populations; for concomitant scleritis and uveitis; for an intraocular granuloma or retinal inflammation; and for poor response to steroids. ${ }^{13}$ All sexual partners should also be screened via a specialized sexual health services. Furthermore, given the shared risk factors, strong association with syphilis reemergence, and possible increased risk of progression to neurosyphilis, HIV coinfection should also be routinely tested. Once diagnosed, it is recommended that all patients with ocular syphilis undergo an LP for cerebrospinal fluid (CSF) analysis to exclude other manifestations of neurosyphilis. ${ }^{20}$

With ocular syphilis representing a manifestation of neurosyphilis, current treatment guidelines advice treatment as per neurosyphilis, with prompt IV penicillin G highly effective in resolving the infection. ${ }^{7,20}$ In the setting of ocular inflammation, adjunctive use of steroids (along with concurrent antibiotic therapy) may be of benefit: topical steroids for interstitial keratitis and anterior uveitis, and systemic steroids for posterior uveitis, scleritis, optic neuritis, and in cases of profound visual loss. . $^{7,21}$

Once treatment is initiated, patients should be followed up clinically and with serial syphilis serology and LPs carried out at regular intervals following treatment: 6, 12, and 24 months. Failure of nontreponemal titer levels to decline fourfold within 6-12 months may be an indication of treatment failure or possible reinfection. ${ }^{22,23}$ Patients with HIV coinfection may display a slower rate of decline and treatment failure may be more common, thereby requiring a more stringent clinical and serological follow-up period $\left(3,6,9,12\right.$, and 24 months). ${ }^{7,22}$ Thus, successful treatment of ocular syphilis results in resolution of ocular inflammation, decline in nontreponemal antibody titers, and normalization of CSF pleocytosis. ${ }^{7,22,23}$ Although the three cases described demonstrated varied visual outcomes, they all received appropriate treatment and showed resolution of syphilis infection by the end of their follow-up period.

\section{Conclusion}

Ocular syphilis can be misdiagnosed at first presentation, owing to the wide range of ocular manifestations. Mirroring worldwide trends, the prevalence of infection in Australia is increasing, particularly in MSM, but also within heterosexual Indigenous Australians. There should be a low threshold to test patients with ocular inflammation for syphilitic disease. If a patient is diagnosed with ocular syphilis, they should be tested for other STIs, including HIV. LP and subsequent CSF analysis should be carried out in all patients with ocular involvement. Treatment follows the same guidelines as per neurosyphilis, and topical ocular and systemic steroids may be warranted depending on the level of ocular involvement. Follow-up is required with repeat nontreponemal serology and LPs for up to 2 years to establish successful treatment.

\section{Disclosure}

The authors report no conflicts of interest in this work.

\section{References}

1. Lee SY, Cheng V, Rodger D, Rao N. Clinical and laboratory characteristics of ocular syphilis: a new face in the era of HIV co-infection. J Ophthalmic Inflamm Infect. 2015;5:26.

2. Communicable Disease Intelligence. National Notifiable Diseases Surveillance System Surveillance Report: Sexually Transmissible Infections in Aboriginal and Torres Straight Islander People, 2015. Available from: http://www.health.gov.au/internet/main/publishing.nsf/Content/ cdi3904-1. Accessed February 25, 2016.

3. The Kirby Institute. HIV, Viral Hepatitis and Sexually Transmissible Infections in Australia Annual Surveillance Report. Sydney, NSW: The University of New South Wales; 2015. Available from: https:// kirby.unsw.edu.au/surveillance/2015-annual-surveillance-report-hivviral-hepatitis-stis. Accessed February 25, 2016.

4. Shen J, Feng L, LiY. Ocular syphilis: an alarming infectious eye disease. Int J Clin Exp Med. 2015;8(5):7770-7777.

5. Ghanem KG, Moore RD, Rompalo AM, Erbelding EJ, Zenilman JM, Gebo KA. Neurosyphilis in a clinical cohort of HIV-1-infected patients. AIDS. 2008;22(10):1145-1151.

6. Hughes EH, Guzowski M, Simunovic MP, Hunyor AP, McCluskey P. Syphilitic retinitis and uveitis in HIV-positive adults. Clin Experiment Ophthalmol. 2010;38(9):851-856.

7. Kiss S, Damico FM, Young LH. Ocular manifestations and treatment of syphilis. Semin Ophthalmol. 2005;20(3):161-167. 
8. Yang P, Zhang N, Li F, Chen Y, Kijlstra A. Ocular manifestations of syphilitic uveitis in Chinese patients. Retina. 2012;32(9):1906-1914.

9. Aldave AJ, King JA, Cunningham ET Jr. Ocular syphilis. Curr Opin Ophthalmol. 2001;12(6):433-441.

10. Mikita C, Trusdell A, Katial RK. A 48-year old woman with red eyes and a rash. Ann Allergy Asthma Immunol. 2004;93(6):526-531.

11. Tamesis RR, Foster CS. Ocular syphilis. Ophthalmol. 1990;97(10): 1281-1287.

12. Tucker JD, Li JZ, Robbins GK, et al. Ocular syphilis among HIVinfected patients: a systematic analysis of the literature. Sex Transm Infect. 2011;87(1):4-8.

13. Gaudio PA. An update on ocular syphilis. Curr Opin Ophthalmol. 2006;17(6):562-566.

14. Browning DJ. Posterior segment manifestations of active ocular syphilis, their response to a neurosyphilis regimen of penicillin therapy, and the influence of human immunodeficiency virus status on response. Ophthalmol. 2000;107(11):2015-2023.

15. Northey LC, Skalicky SE, Gurbaxani A, McCluskey PJ. Syphilitic uveitis and optic neuritis in Sydney, Australia. Br J Ophthalmol. 2015;99(9):1215-1219.
16. Pringle E, Bingham J, Graham E. Progressive binasal hemianopia. Lancet. 2004;363:1606.

17. Wilhelmus KR, Jones DB. Adult-onset syphilitic stromal keratitis. Am J Ophthalmol. 2006;141(2):319-321.

18. Yoon KC, Im SK, Seo MS, Park YG. Neurosyphilitic episcerlitis. Acta Ophthalmol Scand. 2005;83(2):265-266.

19. Tsan GL, Amin P, Sullivan-Mee. Nongranulomatous uveitis as the first manifestation of syphilis. Optom Vis Sci. 2016;93(6):647-651.

20. Centers for Disease Control and Prevention, National Notifiable Diseases Surveillance System. Clinical Advisory: Ocular Syphilis in the United States, 2015. Available from: http://www.cdc.gov/std/syphilis/ clinicaladvisoryos2015.htm. Accessed April 1, 2016.

21. Bollemeijer JG, Wieringa WG, Missotten TO, et al. Clinical manifestations and outcome of syphilitic uveitis. Invest Ophthalmol Vis Sci. 2016;57(2):404-411.

22. Centers for Disease Control and Prevention. 2015 Sexually Transmitted Diseases Treatment Guidelines: Syphilis, 2015. Available from: http:// www.cdc.gov/std/tg2015/syphilis.htm. Accessed January 20, 2016.

23. Zelstser R, Kurban AK. Syphilis. Clin Dermatol. 2004;22(6) 461-468.
International Medical Case Reports Journal

\section{Publish your work in this journal}

The International Medical Case Reports Journal is an international, peer-reviewed open-access journal publishing original case reports from all medical specialties. Previously unpublished medical posters are also accepted relating to any area of clinical or preclinical science. Submissions should not normally exceed 2,000 words or

\section{Dovepress}

4 published pages including figures, diagrams and references. The manuscript management system is completely online and includes a very quick and fair peer-review system, which is all easy to use. Visit http://www.dovepress.com/testimonials.php to read real quotes from published authors. 